\title{
Dispersive charge density wave excitations in $\mathrm{Bi}_{2} \mathrm{Sr}_{2} \mathrm{CaCu}_{2} \mathrm{O}_{8+\delta}$
}

\author{
L. Chaix ${ }^{1}$, G. Ghiringhelli ${ }^{2,3}$, Y. Y. Peng ${ }^{2}$, M. Hashimoto ${ }^{4}$, B. Moritz ${ }^{1}$, K. Kummer ${ }^{5}$, N. B. Brookes ${ }^{5}$, \\ Y. He ${ }^{6}$, S. Chen ${ }^{6}$, S. Ishida ${ }^{7}$, Y. Yoshida ${ }^{7}$, H. Eisaki ${ }^{7}$, M. Salluzzo ${ }^{8}$, L. Braicovich ${ }^{2,3}$, Z.-X. Shen ${ }^{1,6 \star}$, \\ T. P. Devereaux ${ }^{1,6 \star}$ and W.-S. Lee ${ }^{1 \star}$
}

Experimental evidence on high- $T_{c}$ cuprates reveals ubiquitous charge density wave (CDW) modulations ${ }^{1-10}$, which coexist with superconductivity. Although the CDW had been predicted by theory ${ }^{11-13}$, important questions remain about the extent to which the CDW influences lattice and charge degrees of freedom and its characteristics as functions of doping and temperature. These questions are intimately connected to the origin of the CDW and its relation to the mysterious cuprate pseudogap $^{10,14}$. Here, we use ultrahigh-resolution resonant inelastic $X$-ray scattering to reveal new CDW character in underdoped $\mathrm{Bi}_{2.2} \mathrm{Sr}_{1.8} \mathrm{Ca}_{0.8} \mathrm{Dy}_{0.2} \mathrm{Cu}_{2} \mathrm{O}_{8+\delta}$. At low temperature, we observe dispersive excitations from an incommensurate CDW that induces anomalously enhanced phonon intensity, unseen using other techniques. Near the pseudogap temperature $T^{*}$, the CDW persists, but the associated excitations significantly weaken with an indication of CDW wavevector shift. The dispersive CDW excitations, phonon anomaly, and analysis of the CDW wavevector provide a comprehensive momentumspace picture of complex CDW behaviour and point to a closer relationship with the pseudogap state.

With sufficient energy resolution, resonant inelastic X-ray scattering (RIXS) can be an ideal probe for revealing the CDW excitations in cuprates. By tuning the incident photon energy to the $\mathrm{Cu} \mathrm{L}_{3}$-edge (Fig. 1a), the resonant absorption and emission processes can leave the system in excited final states, which couple to a variety of excitations arising from orbital, spin, charge, and lattice degrees of freedom ${ }^{15}$. Thus, information of these elementary excitations in energy and momentum space can be deduced from analysing the RIXS spectra as functions of the energy loss and the momentum transfer of the photons (Fig. 1a). This is highlighted by the pivotal role that RIXS has recently played in revealing orbital and magnetic excitations in cuprates ${ }^{16-20}$. In addition, RIXS provided the first $\mathrm{X}$-ray scattering evidence for an incommensurate $\mathrm{CDW}$ in (Y,Nd) $\mathrm{Ba}_{2} \mathrm{Cu}_{3} \mathrm{O}_{6+\delta}$ (ref. 4), owing to energy resolution that separated the quasi-elastic CDW signal (bright spot in Fig. 1b, limited by the instrumental resolution $\sim 130 \mathrm{meV}$ ) from other intense higher-energy excitations. Notably this quasi-elastic signal is asymmetric with respect to zero energy loss (Fig. 1c), which indicates the possible existence of additional low-energy excitations near the CDW wavevector $\left(Q_{\mathrm{CDW}}\right)$.
In this work, we exploit the newly commissioned ultrahighresolution RIXS instrument at the European Synchrotron Radiation Facility to reveal these low-energy excitations near the CDW. We choose the double-layer cuprate $\mathrm{Bi}_{2.2} \mathrm{Sr}_{1.8} \mathrm{Ca}_{0.8} \mathrm{Dy}_{0.2} \mathrm{Cu}_{2} \mathrm{O}_{8+\delta}$ (Bi2212), whose electronic structure has been extensively studied by surface-sensitive spectroscopy, such as scanning tunnelling microscopy ${ }^{21}$ and angle-resolved photoemission ${ }^{22}$, and in which a short-range CDW order was recently reported ${ }^{7,8}$. With improved energy resolution up to $40 \mathrm{meV}$, we see additional features in the previous quasi-elastic region (Fig. 1d).

Figure 2a presents an energy-momentum RIXS intensity map of our high-resolution data. Two excitation branches are clearly observed with a momentum-dependent intensity distribution, also evident in the energy-loss spectra at representative momenta (Fig. 2b). The first branch centred at zero energy should contain the scattering signal due to an underlying CDW. Indeed, as plotted in Fig. 2c, the momentum distribution of the RIXS intensity averaged over a small energy window exhibits a symmetric peak at a finite momentum, with a peak-to-background ratio of $\sim 2$, unambiguously confirming the existence of the CDW in Bi2212. The peak position is located at $Q_{\mathrm{CDW}} \sim 0.3$ reciprocal lattice units (r.l.u.), consistent with previous STM studies ${ }^{8}$ and with a full-width at half-maximum (FWHM) of approximately 0.085 r.l.u., corresponding to a short correlation length of approximately $15 \AA$ A. Interestingly, despite the short correlation length, the CDW strength, estimated by the integrated intensity of the CDW peak in the quasi-elastic region, is not weaker than that in $\mathrm{YBa}_{2} \mathrm{Cu}_{3} \mathrm{O}_{6+x}$ (see Supplementary Information).

The second branch of excitations possesses an energy scale of approximately $60 \mathrm{meV}$, whose energy-momentum-dispersion can be reliably extracted (Fig. 2d and Supplementary Fig. 2). The extracted dispersion agrees well with that of the $\mathrm{Cu}-\mathrm{O}$ bond-stretching phonon measured by non-resonant inelastic X-ray scattering $^{23}$ (Supplementary Information). Owing to the high momentum-resolution of our data, Fig. 2e shows mode softening by approximately $25 \%$ at $Q_{\mathrm{CDW}}$, with a corresponding broadening of the fitted peak width, indicating that the short-ranged ordered CDW unambiguously affects the lattice. This observation is reminiscent of the bond-stretching phonon anomaly reported in the stripedordered cuprates ${ }^{24}$ and the acoustic phonon anomaly in $\mathrm{YBCO}^{25}$, where CDW correlation lengths are notably longer. Curiously, the

\footnotetext{
${ }^{1}$ Stanford Institute for Materials and Energy Sciences, SLAC National Accelerator Laboratory and Stanford University, 2575 Sand Hill Road, Menlo Park, California 94025, USA. ${ }^{2}$ Dipartimento di Fisica, Politecnico di Milano, Piazza Leonardo da Vinci 32, I-20133 Milano, Italy. ${ }^{3}$ CNR-SPIN, CNISM, Politecnico di Milano, Piazza Leonardo da Vinci 32, I-20133 Milano, Italy. ${ }^{4}$ Stanford Synchrotron Radiation Lightsource, SLAC National Accelerator Laboratory, 2575, Sand Hill Road, Menlo Park, California 94025, USA. ${ }^{5}$ European Synchrotron Radiation Facility (ESRF), BP 220, F-38043 Grenoble Cedex, France. ${ }^{6}$ Geballe Laboratory for Advanced Materials, Stanford University, Stanford, California 94305, USA. ${ }^{7}$ National Institute of Advanced Industrial Science and Technology (AIST), Tsukuba, Ibaraki 305-8560, Japan. ${ }^{8}$ CNR-SPIN, Complesso Monte Sant'angelo, Via Cinthia, I-80126 Napoli, Italy. 
a

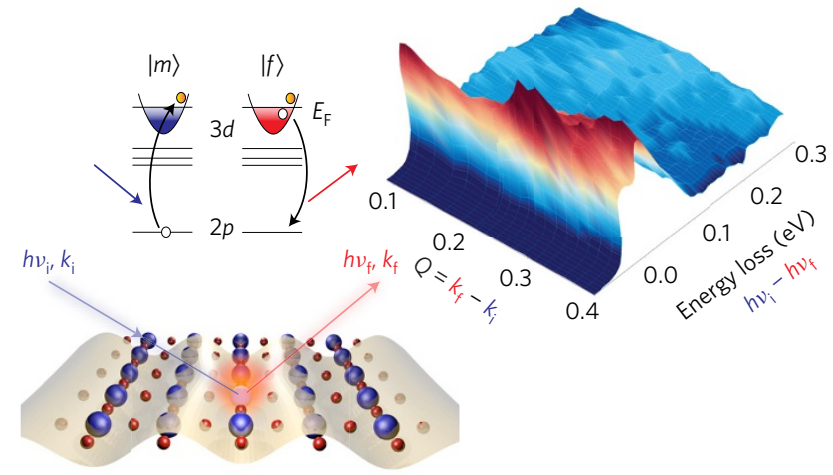

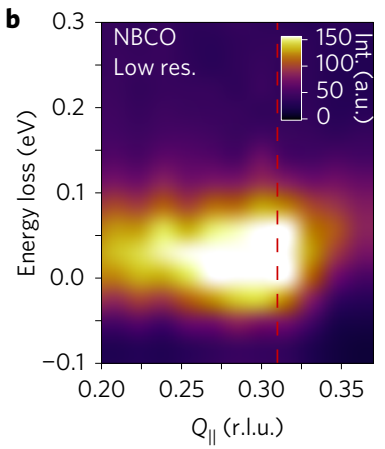
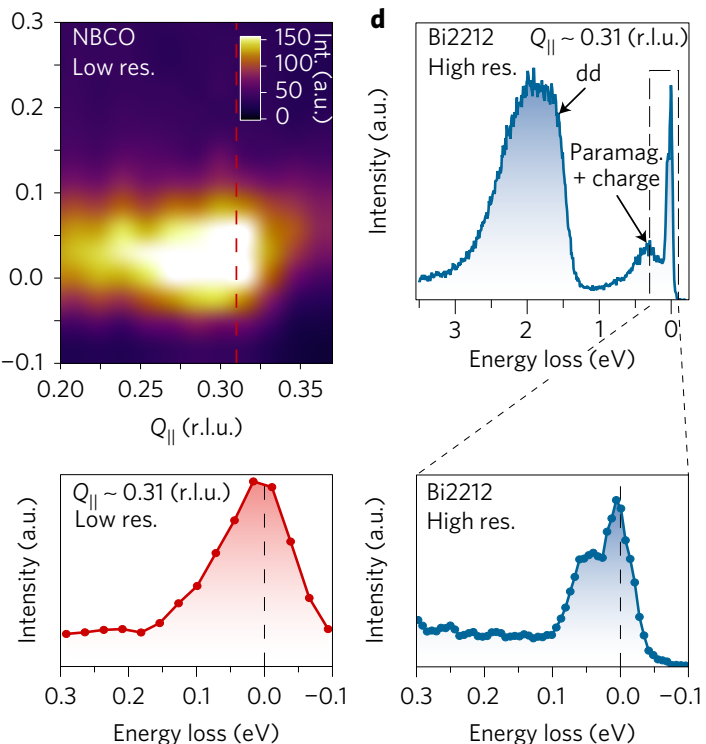

Figure 1 | RIXS process and a hint of lower-energy excitations near $\mathbf{Q}_{\mathrm{CDW}}$. $\mathbf{a}$, Schematics of the coherent two-step RIXS process. The photon energy of the incident $\mathrm{X}$-ray $\left(h v_{\mathrm{i}}\right)$ is tuned to the $\mathrm{Cu} \mathrm{L}_{3}$-edge. Upon absorption, a core electron in the $\mathrm{Cu} 2 \mathrm{p}$ orbital made a transition to the unoccupied $3 d$ valence band near the Fermi energy $E_{\mathrm{F}}$, bringing the system to an intermediate state $|m\rangle$. Then, the emission process occurs that fills the $2 p$ core hole by one of the valence electrons, emits a photon $\left(h \nu_{f}\right)$, and leaves the system in an excited final state $|f\rangle$, which couples to a variety of elementary excitations. The energy-momentum information of these elementary excitations can be deduced by tracking the peak and spectral weight in RIXS spectra as a function of the momentum transfer $Q$ and the energy loss of the scattering photons. The momentum of the incident and scattered photons is represented by $k_{i}$ and $k_{f}$, respectively. Since the electronic state in Bi2212 is quasi-two-directional (that is, almost independent along the $c$-axis), all data are plotted as a function of projected momentum transfer $\mathrm{Q}_{\|}$along the [100] direction (that is, along the $\mathrm{Cu}-\mathrm{O}$ bond direction). $\mathbf{b}$, RIXS intensity map of the underdoped $\mathrm{Nd}_{1.2} \mathrm{Ba}_{1.8} \mathrm{Cu}_{3} \mathrm{O}_{6+\delta}$ (UD-NBCO) compound around the CDW position, taken with a resolution of $130 \mathrm{meV}$. c, RIXS spectrum along the red dashed line shown in b. d, A high energy-resolution ( $40 \mathrm{meV}$ ) RIXS spectrum of UD-Bi2212 at $Q_{\|}=0.31$ r.l.u. An enlarged view of the black-dashed box near the elastic peak is shown in the lower panel.
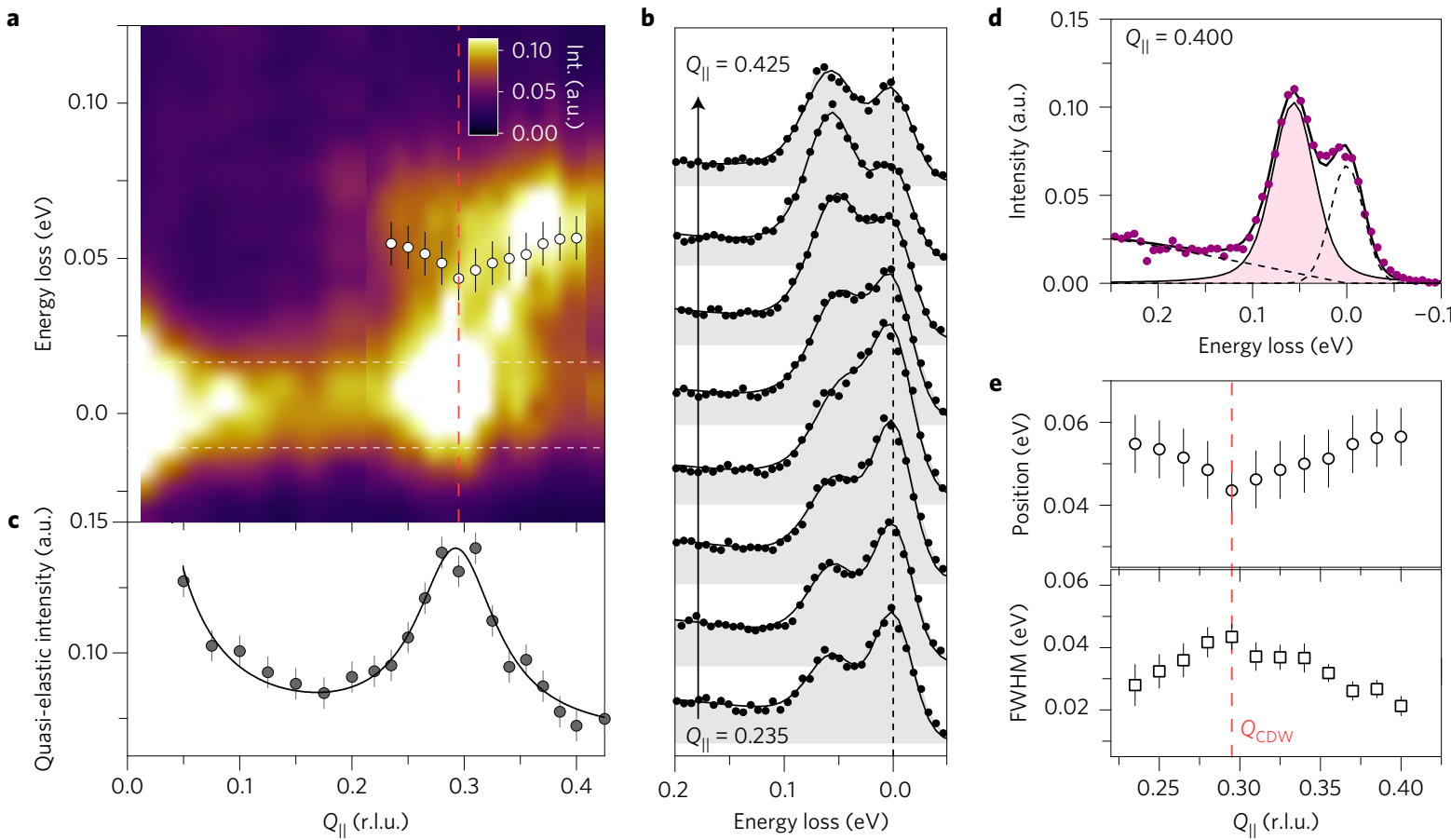

Figure 2 | CDW and phonons at $20 \mathrm{~K}$. a, RIXS intensity map of the high energy-resolution data ( $\triangle E \sim 40 \mathrm{meV}$ ) as a function of energy loss and $\mathrm{Q}_{\|}$. The white circles represent the fitted phonon dispersion. b. Energy-loss spectra at selected momentum values ranging from $Q_{\|}=0.235$ r.l.u. to $Q_{\|}=0.425$ r.l.u. The fits (solid lines) are superimposed on the raw data (black circles). c, Averaged intensity in the quasi-elastic region, defined as the region between the two white dashed lines in $\mathbf{a}$. The solid line is a Lorentzian fit to the data with a background consisting of a constant plus a Lorentzian to account for the tail of the specular reflection peak at $Q_{\|}=0$. d, Demonstration of the quality of the fit of a RIXS spectrum at a representative momentum. The fitting process is described in the Methods section. The phonon peak is highlighted in pink. e, Position and FWHM (de-convolved) of the measured phonon peak extracted from the fits. The red dashed lines on $\mathbf{a}$ and $\mathbf{e}$ indicate $Q_{C D W}$. Error bars in $\mathbf{a}$ and $\mathbf{e}$ (upper panel) are estimated by the uncertainty in determining the zero energy loss. The error bars for the FWHM in e are two standard deviations from the fit. Those in $\mathbf{c}$ are determined by the noise level of the spectra. 
$\mathrm{Bi}-2212$

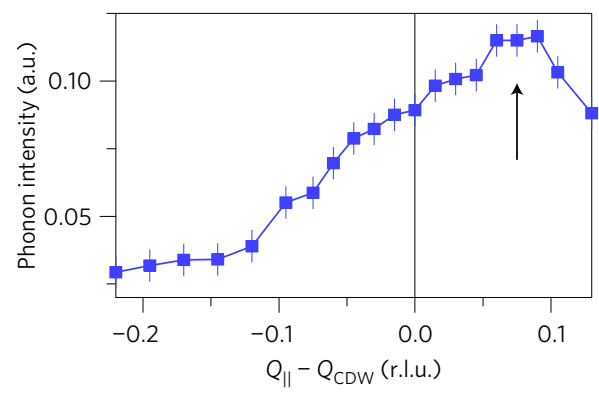

d

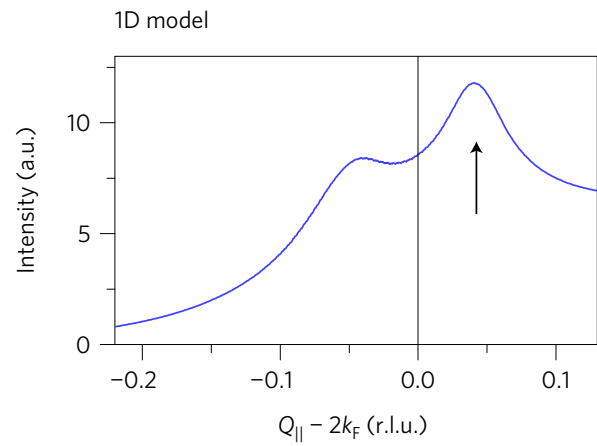

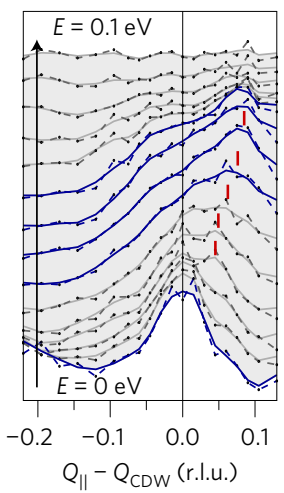

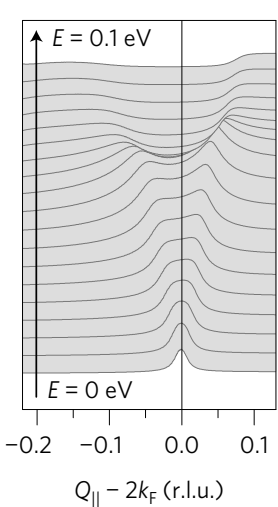

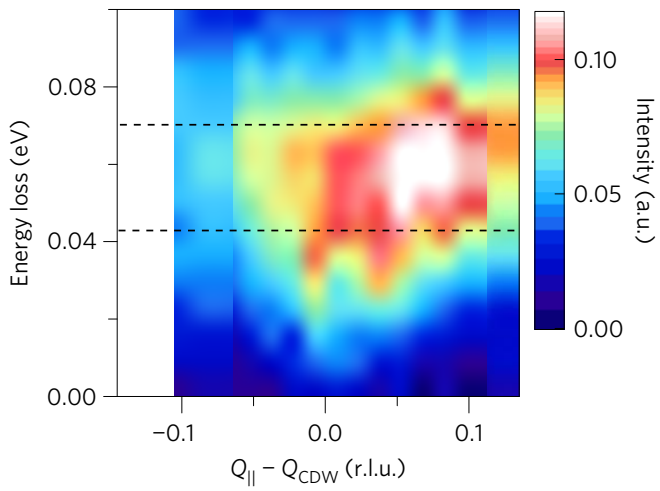

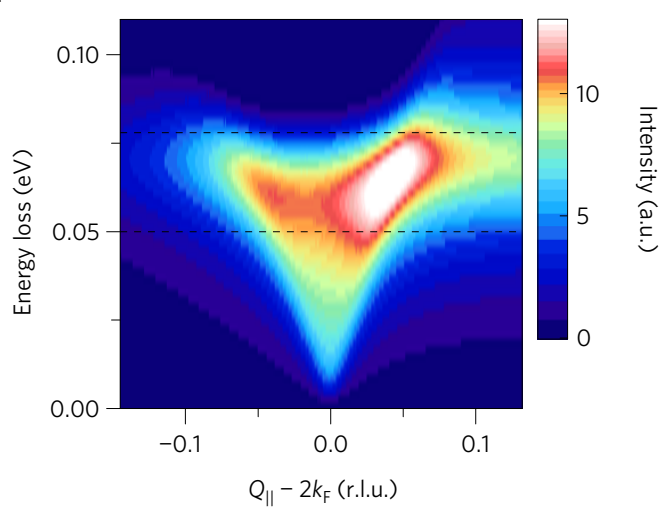

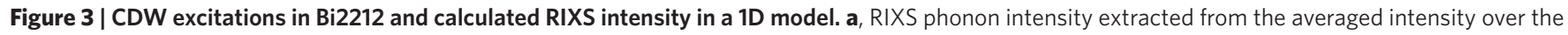
energy range between the two black-dashed lines in c. The black arrow highlights the intensity anomaly $Q_{A}$. Error bars are estimated by the noise level of the spectra. b. Momentum-distribution curves (MDCs) at fixed energy of the raw data (Fig. $2 a$ ) from $E=0$ eV to $E=0.1$ eV. Both the raw data (markers with dashed lines) and the smoothed curves (solid lines) are superimposed. The MDCs corresponding to the CDW and phonon energy regions are highlighted in blue. The red ticks serve as guides to the eye for the connection between CDW and the phonon intensity anomaly. QCDW represents the CDW wavevector. c, Elastic peak-subtracted intensity map. $\mathbf{d}-\mathbf{f}$, Calculations of RIXS phonon intensity for a 1D metallic system, with the same plotting format and notations used in a-c. $2 k_{F}$ represents the perfect nesting wavevector of the 1D system. All the experimental (calculated) data are plotted as a function of $Q_{\|}-Q_{C D W}\left(Q_{\|}-2 k_{F}\right)$.

phonon softening occurs gradually near $Q_{\mathrm{CDW}}$, unlike the Kohn anomaly due to the reduced lattice symmetry from the CDW, which usually occurs more abruptly in a narrow momentum window. In addition, whereas the width of the phonon self-energy directly measured by inelastic neutron or non-resonant inelastic X-ray scattering is typically a few millielectronvolts ${ }^{23,24}$, the fitted width of peak shown in Fig. 2e progressively broadens near $Q_{\mathrm{CDW}}$ and is not resolution limited $(\sim 40 \mathrm{meV})$, hinting at the existence of additional low-energy excitations.

Remarkably, the phonon intensity varies non-monotonically with momentum, having a maximum near $Q_{\mathrm{A}} \sim 0.37$ r.l.u $=0.07$ r.l.u. + $Q_{\mathrm{CDW}}$ (Fig. 3a). It is important to note that the RIXS phonon crosssection directly reflects the momentum dependence of the electronphonon coupling strength ${ }^{26-28}$ (Supplementary Information), in stark contrast with the phonon self-energy measured by other scattering techniques. As a consequence, RIXS is also directly sensitive to the interference between phonons and underlying charge excitations (that is, the Fano effect), which can manifest as an intensity anomaly. Thus, the intensity anomaly at $Q_{\mathrm{A}} \neq Q_{\mathrm{CDW}}$ (Figs 2a and 3a) provides evidence for the existence of dispersive CDW excitations that cause a strong Fano interference at the intersection $Q_{\mathrm{A}}$ with the bond-stretching phonon.

Subtle but non-negligible spectral features further support the existence of dispersive CDW excitations. Examining the momentum-distribution curves (Fig. 3b), the peak at zero energy disperses and broadens with increasing energy loss, smoothly connecting to the phonon at $Q_{\mathrm{A}}$. To visualize this connection in an alternative way, we remove the elastic contribution from the energy-loss spectra (Fig. 3c) to reveal a funnel-shaped RIXS intensity emanating from $Q_{\mathrm{CDW}}$, suggesting that these excitations are associated with the CDW. This funnel-shaped intensity essentially reflects the anomalous broadening of the peak width near $Q_{\mathrm{CDW}}$ shown in Fig. 2e. These CDW excitations disperse to high energy and intersect with the phonon at $Q_{\mathrm{A}}$, causing the phonon intensity anomaly due to the strong Fano interference effect.

To demonstrate the Fano resonance in the RIXS phonon crosssection, we calculate the RIXS intensity map for a one-dimensional (1D) metallic system with coupling between electrons and a bondstretching phonon mode (Fig. 3f). Perfect Fermi surface nesting at $2 k_{\mathrm{F}}$ (that is, twice the Fermi momentum) creates a particlehole continuum, whose presence softens the phonon dispersion (that is, not a Kohn-like phonon anomaly) and creates an intensity anomaly at the intersection between the continuum and the phonon (Fig. 3d-f). This result qualitatively agrees with our observations in Bi2212. We also note that the strength of the Fano interference is dictated by the electron-phonon coupling strength at the intersecting momentum. Since the coupling strength of the bondstretching phonon is zero at $Q=0$ and increases monotonically with momentum, the Fano interference is weaker at $Q<Q_{\mathrm{CDW}}$ than at $Q>Q_{\mathrm{CDW}}$. Finally, by connecting $Q_{\mathrm{CDW}}$ and $Q_{\mathrm{A}}$, we deduce a characteristic velocity of these CDW excitations: $V_{\mathrm{CDW}} \sim 0.6 \pm 0.2 \mathrm{eV} \AA$. We find that $V_{\mathrm{CDW}}$ is neither equivalent to the Fermi velocity seen in ARPES nor the quasi-particle-interference dispersion seen in $\mathrm{STM}^{21}$ (Supplementary Information), indicating that the weak-coupling fermiology may be irrelevant for describing the observed dispersion. 

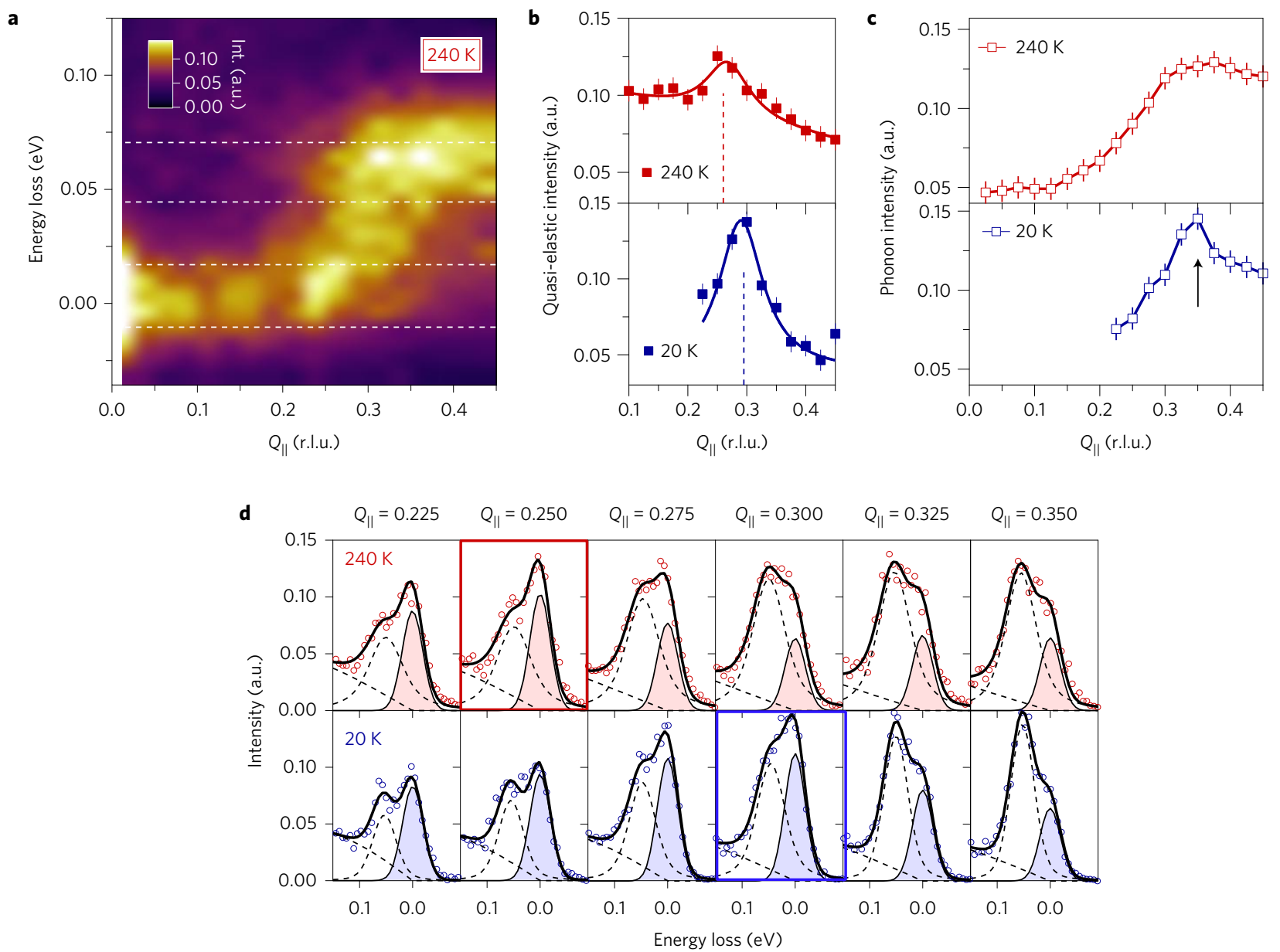

Figure 4 | Temperature dependence of the CDW and the phonon anomaly. $\mathbf{a}, \mathrm{RIXS}$ intensity map taken at $T=240 \mathrm{~K}$. The white dashed lines define the quasi-elastic region (centred at zero energy loss) and the phonon energy region (centred at $60 \mathrm{meV}$ ) used for calculating the averaged intensity profile shown in $\mathbf{b}$ and $\mathbf{c}$. $\mathbf{b}$, Averaged intensity of the quasi-elastic region (indicated in $\mathbf{a}$ ) at $240 \mathrm{~K}$ (red) and $20 \mathrm{~K}$ (blue). The dashed lines indicate the position of the CDW. The RIXS intensity map taken at $T=20 \mathrm{~K}$ is presented in Supplementary Fig. 4. c, Averaged intensity of the phonon energy regions, as defined in $\mathbf{a}$, at $240 \mathrm{~K}$ and $20 \mathrm{~K}$. The black arrow highlights the intensity anomaly at $20 \mathrm{~K}$. Error bars in $\mathbf{b}$ and $\mathbf{c}$ are estimated by the noise level of the spectra. $\mathbf{d}$, Raw energy-loss spectra (markers) and the corresponding fits (solid lines) of RIXS data taken at $T=240 \mathrm{~K}$ (top) and $T=20 \mathrm{~K}$ (bottom). The elastic peak fits are highlighted with filled areas. The RIXS spectra where the elastic peak intensity is maximal (that is, the CDW position) are indicated by red and blue boxes for $T=240 \mathrm{~K}$ and $T=20 \mathrm{~K}$, respectively.

Temperature-dependent measurements were conducted on another Bi2212 crystal with a similar $T_{\mathrm{c}}(40 \mathrm{~K})$, with results shown in Fig. 4. We first confirm that the low-temperature measurement at $20 \mathrm{~K}$ reproduces all the observations shown in Fig. 2 (Supplementary Fig. 4). Upon increasing the temperature to $240 \mathrm{~K}$ (near the pseudogap temperature ${ }^{22}$ ), we find that the CDW signal in the quasi-elastic region persists (Fig. 4a) with reduced peak intensity (Fig. 4b). We also observed that whereas the phonon softening still remains (Supplementary Fig. 4), the intensity anomaly at $Q_{\mathrm{A}}$ diminishes and broadens, as shown by the momentum distribution of the phonon intensity in Fig. 4c. This indicates that the CDW excitations still notably affect the charge dynamics, but become poorly defined in energymomentum space as the $\mathrm{CDW}$ weakens. Interestingly, $Q_{\mathrm{CDW}}$ appears to change from incommensurate 0.3 r.l.u. at $20 \mathrm{~K}$ to nearly commensurate $\sim 0.26$ r.l.u. at $240 \mathrm{~K}$ (Fig. 4 b,d), which corresponds to a real-space periodicity of approximately four lattice constants $\left(\sim 4 a_{0}\right)$, similar to the stripe-ordered cuprates ${ }^{1,3}$. This indication of temperature-dependent $\mathrm{CDW}$ commensuration requires future investigation to further confirm these findings.

Our results have several important implications. They provide the first direct evidence for the existence of dispersive CDW excitations in the charge-charge correlation function with a characteristic velocity $V_{\mathrm{CDW}}$. Furthermore, these excitations persist to high energy, at least up to $\sim 60 \mathrm{meV}$, causing the phonon intensity anomaly, indicating that the CDW influences the charge and lattice degrees of freedom, despite its short correlation length. Whether the observed CDW excitations are collective due to a symmetry breaking ${ }^{29}$ or rather a reflection of the particle-hole continuum remains an open question for future investigation. Additionally, if confirmed, the surprising change of $Q_{\mathrm{CDW}}$ from incommensurate at low temperature towards commensurate at high temperature is reminiscent of the temperature dependence observed in stripe-phased nickelates, where the charge order is strongly coupled to a spin order, which originates from strong real-space interactions $\mathrm{s}^{30}$. These results may be consistent with the proposal that the incommensurate $Q_{\mathrm{CDW}}$ forms from a discommensuration of a universal period $4 a_{0}$ modulations ${ }^{31}$. Finally, the CDW signatures survive up to at least $240 \mathrm{~K} \sim T^{*}$, which taken together with the similarities to striped nickelates indicates a close relationship between the CDW, spins, and the pseudogap.

\section{Methods}

Methods, including statements of data availability and any associated accession codes and references, are available in the online version of this paper. 
Received 9 January 2017; accepted 3 May 2017; published online 12 June 2017

\section{References}

1. Tranquada, J. M., Sternlieb, B. J., Axe, J. D., Nakamura, Y. \& Uchida, S. Evidence for stripe correlations of spins and holes in copper oxide superconductors. Nature 375, 561-563 (1995).

2. Howald, C., Eisaki, H., Kaneko, N. \& Kapitulnik, A. Coexistence of periodic modulation of quasiparticle states and superconductivity in $\mathrm{Bi}_{2} \mathrm{Sr}_{2} \mathrm{CaCu}_{2} \mathrm{O}_{8+\delta}$. Proc. Natl Acad. Sci. USA 100, 9705-9709 (2003).

3. Abbamonte, P. et al. Spatially modulated 'Mottness' in $\mathrm{La}_{2-x} \mathrm{Ba}_{x} \mathrm{CuO}_{4}$. Nat. Phys. 1, 155-158 (2005).

4. Ghiringhelli, G. et al. Long-range incommensurate charge fluctuations in ( $\mathrm{Y}, \mathrm{Nd}) \mathrm{Ba}_{2} \mathrm{Cu}_{3} \mathrm{O}_{6+x}$. Science 337, 821-825 (2012).

5. Chang, J. et al. Direct observation of competition between superconductivity and charge density wave order in $\mathrm{YBa}_{2} \mathrm{Cu}_{3} \mathrm{O}_{667}$. Nat. Phys. 8, 871-876 (2012).

6. Comin, R. et al. Charge order driven by Fermi-arc instability in $\mathrm{Bi}_{2} \mathrm{Sr}_{2-x} \mathrm{La}_{x} \mathrm{CuO}_{6+\delta}$. Science 343, 390-392 (2014).

7. Hashimoto, M. et al. Direct observation of bulk charge modulations in optimally doped $\mathrm{Bi}_{15} \mathrm{~Pb}_{06} \mathrm{Sr}_{154} \mathrm{CaCu}_{2} \mathrm{O}_{8+s}$. Phys. Rev. B 89, 220511(R) (2014).

8. da Silva Neto, E. H. et al. Ubiquitous interplay between charge ordering and high-temperature superconductivity in cuprates. Science 343, 393-396 (2014).

9. Tabis, W. et al. Charge order and its connection with Fermi-liquid charge transport in a pristine high- $T_{\mathrm{c}}$ cuprate. Nat. Commun. 5, 5875 (2014).

10. Comin, R. \& Damascelli, A. Resonant X-ray scattering studies of charge order in cuprates. Annu. Rev. Condens. Matter Phys. 7, 369-405 (2016).

11. Zaanen, J. \& Gunnarsson, O. Charged magnetic domain lines and the magnetism of high- $T_{\mathrm{c}}$ oxides. Phys. Rev. B 40, 7391(R) (1989).

12. Poilblanc, D. \& Rice, T. M. Charged solitons in the Hartree-Fock approximation to the large-U Hubbard model. Phys. Rev. B 39, 9749(R) (1989).

13. Emery, V. J., Kivelson, S. A. \& Lin, H. Q. Phase separation in the $t-J$ model. Phys. Rev. Lett. 64, 475-478 (1990).

14. Badoux, S. et al. Change of carrier density at the pseudogap critical point of a cuprate superconductor. Nature 531, 210-214 (2016).

15. Ament, L. J. P., van Veenendaal, M., Devereaux, T. P., Hill, J. P. \& van den Brink, J. Resonant inelastic X-ray scattering studies of elementary excitations. Rev. Mod. Phys. 83, 705-767 (2011).

16. Braicovich, L. et al. Magnetic excitations and phase separation in the underdoped $\mathrm{La}_{2-x} \mathrm{Sr}_{x} \mathrm{CuO}_{4}$ superconductor measured by resonant inelastic X-ray scattering. Phys. Rev. Lett. 104, 077002 (2010).

17. Le Tacon, M. et al. Intense paramagnon excitations in a large family of high-temperature superconductors. Nat. Phys. 7, 725-730 (2011).

18. Schlappa, J. et al. Spin-orbital separation in the quasi-one-dimensional Mott insulator $\mathrm{Sr}_{2} \mathrm{CuO}_{3}$. Nature 485, 82-85 (2012).

19. Dean, M. P. M. et al. Persistence of magnetic excitations in $\mathrm{La}_{2-x} \mathrm{Sr}_{x} \mathrm{CuO}_{4}$ from the undoped insulator to the heavily overdoped non-superconducting metal. Nat. Mater. 12, 1019-1023 (2013).

20. Peng, Y. Y. et al. Magnetic excitations and phonons simultaneously studied by resonant inelastic X-ray scattering in optimally doped $\mathrm{Bi}_{15} \mathrm{~Pb}_{055} \mathrm{Sr}_{16} \mathrm{La}_{04} \mathrm{CuO}_{6+8}$. Phys. Rev. B 92, 064517 (2015).

21. Schmidt, A. R. et al. Electronic structure of the cuprate superconducting and pseudogap phases from spectroscopic imaging STM. New J. Phys. 13, 065014 (2011).

22. Hashimoto, M., Vishik, I. M., He, R. H., Devereaux, T. P. \& Shen, Z. X. Energy gaps in high-transition-temperature cuprate superconductors. Nat. Phys. 10, 483-495 (2014).
23. Graf, J. et al. Bond stretching phonon softening and kinks in the angle-resolved photoemission spectra of optimally doped $\mathrm{Bi}_{2} \mathrm{Sr}_{1.6} \mathrm{La}_{0.4} \mathrm{Cu}_{2} \mathrm{O}_{6+\delta}$ superconductors. Phys. Rev. Lett. 100, 227002 (2008).

24. Reznik, D. et al. Electron-phonon coupling reflecting dynamic charge inhomogeneity in copper oxide superconductors. Nature 440, $1170-1173$ (2006)

25. Le Tacon, $\mathrm{M}$. et al. Inelastic X-ray scattering in $\mathrm{YBa}_{2} \mathrm{Cu}_{3} \mathrm{O}_{6.6}$ reveals giant phonon anomalies and elastic central peak due to charge-density-wave formation. Nat. Phys. 10, 52-58 (2014).

26. Ament, L. J. P., van Veenendaal, M. \& van den Brink, J. Determining the electron-phonon coupling strength from resonant inelastic X-ray scattering at transition metal L-edges. Euro. Phys. Lett. 95, 27008 (2011).

27. Lee, W. S. et al. Role of lattice coupling in establishing electronic and magnetic properties in quasi-one-dimensional cuprates. Phys. Rev. Lett. 110, 265502 (2013).

28. Devereaux, T. P. et al. Directly characterizing the relative strength and momentum dependence of electron-phonon coupling using resonant inelastic X-ray scattering. Phys. Rev. X 6, 041019 (2016).

29. Kaneshita, E., Ichioka, M. \& Machida, K. Spin and charge excitations in incommensurate spin density wave. J. Phys. Soc. Jpn 70, 866-876 (2001).

30. Kajimoto, R. et al. Hole concentration dependence of the ordering process of the stripe order in $\mathrm{La}_{2-x} \mathrm{Sr}_{x} \mathrm{NiO}_{4}$. Phys. Rev. B 64, 144432 (2001).

31. Mesaros, A. et al. Commensurate $4 a_{0}$-period charge density modulations throughout the $\mathrm{Bi}_{2} \mathrm{Sr}_{2} \mathrm{CaCu}_{2} \mathrm{O}_{8+x}$ pseudogap regime. Proc. Natl Acad. Sci. USA 113, 12661-12666 (2016).

\section{Acknowledgements}

We thank S. A. Kivelson for discussions. This work is supported by the US Department of Energy (DOE), Office of Science, Basic Energy Sciences, Materials Sciences and Engineering Division, under contract DE-AC02-76SF00515. L.C. acknowledges the support from Department of Energy, SLAC Laboratory Directed Research and Development funder contract under DE-AC02-76SF00515. The data in Fig. 1b were taken partly at the Advanced Resonant Spectroscopies (ADRESS) beam line of the Swiss Light Source, using the Super Advanced X-ray Emission Spectrometer (SAXES) instrument jointly built by Paul Scherrer Institut (Villigen, Switzerland), Politecnico d Milano (Italy), and École Polytechnique Fédérale de Lausanne (Switzerland); all other RIXS data were taken at the ID32 of the ESRF (Grenoble, France) using the ERIXS spectrometer designed jointly by the ESRF and Politecnico di Milano. ARPES data were taken at Stanford Synchrotron Radiation Lightsource, operated by the US Department of Energy, Office of Science, Office of Basic Energy Sciences.

\section{Author contributions}

W.-S.L., G.G., L.B., T.P.D. and Z.-X.S. conceived the experiment. L.C., W.-S.L., G.G. Y.Y.P., M.H., L.B., K.K. and N.B.B. conducted the experiment at ESRF. L.C., W.-S.L., G.G., Y.Y.P., L.B. and M.H. analysed the data. T.P.D. and B.M. performed the theoretical calculations. Y.H., S.C., S.I., Y.Y., H.E. and M.S. synthesized and prepared samples for the experiments. L.C. and W.-S.L. wrote the manuscript with input from all the authors.

\section{Additional information}

Supplementary information is available in the online version of the paper. Reprints and permissions information is available online at www.nature.com/reprints. Publisher's note: Springer Nature remains neutral with regard to jurisdictional claims in published maps and institutional affiliations. Correspondence and requests for materials should be addressed to Z.-X.S., T.P.D. or W.-S.L.

\section{Competing financial interests}

The authors declare no competing financial interests. 


\section{Methods}

Sample growth, preparation and characterization. The high-quality UD-Bi2212 $\left(\mathrm{Bi}_{2.2} \mathrm{Sr}_{1.8} \mathrm{Ca}_{0.8} \mathrm{Dy}_{0.2} \mathrm{Cu}_{2} \mathrm{O}_{8+\delta}\right)$ single crystals (Supplementary Fig. 1a, sample 1 and 2) were grown by floating-zone (FZ) methods and annealed in a nitrogen atmosphere. The samples were characterized and roughly aligned using Laue diffraction prior to RIXS measurements (Supplementary Fig. 1a). The samples were cleaved in air immediately before loading into the high-vacuum measurement chamber. The transition temperatures $T_{\mathrm{C}}$ are $45 \mathrm{~K}$ and $40 \mathrm{~K}$ for samples 1 and 2 , respectively (Supplementary Fig. 1b), corresponding to doping concentration $p \sim 8-9 \%$. The X-ray absorption curves for samples 1 and 2 are shown in Supplementary Fig. 1c, showing no apparent contamination. Data shown in Figs 2 and 3 were taken on sample 1. Data in Fig. 4 were taken on sample 2.

Low-resolution RIXS measurements on UD-NBCO. The low energy-resolution RIXS data on the UD-NBCO $\left(\mathrm{Nd}_{1.2} \mathrm{Ba}_{1.8} \mathrm{Cu}_{3} \mathrm{O}_{6+\delta}\right)$ sample were taken at the ADRESS beamline of the Swiss Light Source at the Paul Scherrer Institut (PSI, Switzerland) using the SAXES spectrometer ${ }^{32,33}$. The RIXS spectra were measured at $T=60 \mathrm{~K}$, just below $T_{\mathrm{c}}=65 \mathrm{~K}$ to maximize the CDW peak intensity, and using linear vertical $(\sigma)$ polarized incident photons. The total energy resolution was $\sim 130 \mathrm{meV}$, the scattering angle was $130^{\circ}$. The RIXS map is obtained by combining spectra measured every $2^{\circ}$ in the rotation of the incidence angle on the sample surface. The NBCO films, $100 \mathrm{~nm}$ in thickness, were deposited by high-oxygen-pressure diode sputtering on $\mathrm{SrTiO}_{3}$ (100) single crystals. The lattice parameters measured by X-ray diffraction are $a=b=3.84 \AA$ and $c=11.7 \AA$.

\section{Ultrahigh-resolution RIXS measurements on UD-Bi2212. The RIXS} measurements on the UD-Bi2212 samples 1 and 2 were performed using the ERIXS spectrometer at the ID32 beamline of the European Synchrotron Radiation Facility (ESRF, France). The RIXS spectra were taken with the photon energy of the incident X-rays tuned to the maximum of the absorption curve near the $\mathrm{Cu}$ $\mathrm{L}_{3}$-edge (Supplementary Fig. 1c). The scattering geometry is sketched in Supplementary Fig. 1d. The data were collected at $T=20 \mathrm{~K}$ for sample 1 and $T=20 \mathrm{~K} / 240 \mathrm{~K}$ for sample 2 with a linear vertical polarization ( $\sigma$-polarization) of the incident beam. The energy resolution was approximately $\Delta E \sim 40 \mathrm{meV}$ (sample 1) and $\Delta E \sim 45 \mathrm{meV}$ (sample 2). The scattering angle of the endstation was set at $2 \theta=149.5^{\circ}$. Since the electronic state in Bi2212 is quasi-two-directional (that is, almost independent along the $c$-axis), the data shown in this report are plotted as a function of projected momentum transfer $Q_{\|}$(that is, projection of $Q=k_{\mathrm{f}}-k_{\mathrm{i}}$ on the $\mathrm{CuO}_{2}$ plane). Different in-plane momentum transfers, $Q_{\|}$ (projection of the scattering vector $Q$ along [100]), were obtained by rotating the samples around the vertical $b$-axis. Note that the scattering vector $Q$ is denoted using the pseudotetragonal unit cell with $a=b=3.82 \AA$ and $c=30.84 \AA$, where the $c$-axis is normal to the sample surface. In our convention, positive $Q_{\|}$corresponds to grazing-emission geometry and negative $Q_{\|}$corresponds to grazing-incidence geometry. Supplementary Tables 1 and 2 summarize the $L$ values (that is, projection of the scattering vector $Q$ along [001]) for each momentum in the data of sample 1 and 2 , respectively.

Data analysis and fitting. The ultrahigh-resolution data (UD-Bi2212) were normalized to $I_{0}$ and corrected for self-absorption effects using the formalism described in the Supplementary Methods of ref. 34. The zero energy positions were determined by comparing the spectrum recorded on a small amount of silver paint (at each $Q_{\|}$) near the sample surface and fine adjusted by the fitted elastic peak position. The fitting model involves a Gaussian (elastic peak), a Lorentzian (phonon peak) and a background that fits an anti-symmetrized Lorentzian to the paramagnon at high energy loss. The model is convolved with the energy resolution (Gaussian convolution) and fitted to the data. The results of these fits are presented in Supplementary Fig. 2 for sample $1(T=20 \mathrm{~K})$ and Fig. $4 \mathrm{~d}$ and Supplementary Fig. 3 for sample $2(T=20 \mathrm{~K}$ and $T=240 \mathrm{~K}$ ). Please note the plotted peaks are also already convolved with the energy resolution for ease of perceiving the fit quality.

From this analysis, we extracted the dispersion (peak position) and FWHM (resolution de-convolved) of the phonon peak at $T=20 \mathrm{~K}$ in sample 1 (Fig. 2e) and at $T=20 \mathrm{~K}$ and $T=240 \mathrm{~K}$ in sample 2 (Supplementary Fig. 4c,d). The dispersions and FWHM found at $T=20 \mathrm{~K}$ for both sample 1 and sample 2 are similar, with the presence of a softening and a broadening around the CDW wavevector $\left(Q_{\mathrm{CDW}} \sim 0.3\right.$ r.l.u.). The reproducibility of the low-temperature data in two independent measurements and samples confirms our observations of the CDW and the phonon intensity anomaly due to Fano interference (Supplementary Fig. 4a). In addition, we note that the phonon intensity extracted from the fitting procedure agrees with the averaged intensity around the phonon energy (Supplementary Fig. 5b).

Theory. The calculations in Fig. 3d-f were carried out using a recent framework for phonon contributions to RIXS given in ref. 28. Details are given in that reference. The theory developed in ref. 28 considered lowest-order diagrams for RIXS in the weak electron-phonon limit, giving the first contribution to RIXS coming from the emission of one phonon. The strength of the signal is directly tied to the momentum-dependent electron-phonon coupling. In the limit of small coupling, the intensity smoothly varies in momentum space, with a position that closely follows the bare phonon dispersion. However, if the phonon dispersion crosses the electron-hole continuum, a Fano effect occurs, leading to a strong interference between the dispersions of the two excitations, as shown in Fig. 3d-f.

Since we have used a simple 1D metallic model having a Peierls instability at $2 k_{\mathrm{F}}$, the particle-hole excitations disperse in energy and momentum in a cone from zero energy, with a dispersion set by the Fermi velocity. When the cone of charge excitations crosses the phonon line, an interference occurs that exploits the momentum-dependent electron-phonon coupling $g(q)$. For the case of the bond-stretching phonon, $g(q)$ determined from the nature of deformation coupling between $\mathrm{Cu}$ and $\mathrm{O}$ in-plane displacements is largest for large momentum transfers. Therefore, the intensity is stronger on the higher-momentum side of the intersection of the phonon line with the charge excitations. This effect reproduces the experimental observations well.

Data availability. The data that support the plots within this paper and other findings of this study are available from the corresponding authors upon reasonable request.

\section{References}

32. Ghiringhelli, G. et al. SAXES, a high resolution spectrometer for resonant X-ray emission in the 400-1600 eV energy range. Rev. Sci. Instrum. 77, 113108 (2006).

33. Strocov, V. N. et al. High-resolution soft X-ray beamline ADRESS at the Swiss Light Source for resonant inelastic X-ray scattering and angle-resolved photoelectron spectroscopies. J. Synchrotron Radiat. 17, 631-643 (2010).

34. Minola, M. et al. Collective nature of spin excitations in superconducting cuprates probed by resonant inelastic x-ray scattering. Phys. Rev. Lett. 114, 217003 (2015). 